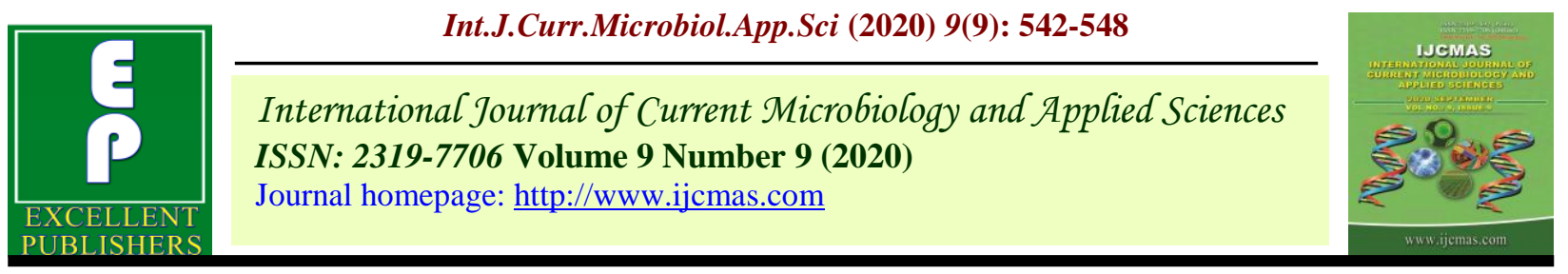

Original Research Article

https://doi.org/10.20546/ijcmas.2020.909.069

\title{
Methicillin Resistant Staphylococcus epidermidis in Blood Samples-Can its Spread be Prevented in Hospitals?
}

\author{
Maryam J. Ansari, Rachna Tewari* and Mridu Dudeja \\ Department of Microbiology, Hamdard Institute of Medical Sciences and Research \\ Jamia Hamdard, New Delhi, India \\ *Corresponding author
}

\section{A B S T R A C T}

\begin{tabular}{l} 
K e y w o r d s \\
$\begin{array}{l}\text { Staphylococcus } \\
\text { epidermidis, } \\
\text { Methicillin } \\
\text { resistance, } \\
\text { Nosocomial } \\
\text { infections, Biofilm } \\
\text { production }\end{array}$ \\
$\begin{array}{l}\text { Article Info } \\
\text { Accepted: } \\
\text { 07 August } 2020 \\
\text { Available Online: } \\
10 \text { September } 2020\end{array}$ \\
\hline
\end{tabular}

Keywords

Staphylococcus

epidermidis,

resistance,

Nosocomial

infections, Biofilm

production

\section{Introduction}

Coagulase-negative cocci, Staphylococcus epidermidis are considered important for two reasons. First, they are now emerged as one of the leading cause of hospital acquired infections. around prosthetic devices, shunts, catheters, and in-dwelling intravascular lines. Second, these organisms are found to be the frequent cause of positive blood cultures in the hospitals as they are the extremely common contaminants of the skin flora. ${ }^{1}$
S.epidermidis previously dismissed as contaminants is now emerged as one of the major nosocomial pathogens exhibiting resistance to various drugs including Methicillin. Their ability to form biofilms contributes to their pathogenicity contributing to increased drug resistance. Proper infection control services can certainly bring the rates of this virulent bacteria down in hospitals. The study was conducted for a period of 6 months (Febrauary 9 to July 2019). S.epidermidis was isolated from blood samples received in chology laboratory and were identified by standard protocol. Antibiotic susceptibility was determined by Kirby -Bauer disc diffusion method and Vitek 2 system\& results were subjected a the total 96 CoNS isolated, 50 were identified as S.epidermidis. Among these S.epidermidis 24 were found to be methicillin resistant and out of these 24 MRSE, 10 isolates were biofilm producers. From the study, we found that most of the strains were collected from IPD patients than OPD patients. So spread of these resistant strains can easily be controlled by proper hospital infection control system. 
methicillin, which is even higher than the corresponding rate for $S$. aureus (40-60\%). Thus, MRSE strains are considered reservoirs of antimicrobial resistance genes that can be transferred to other staphylococci.

This high level of resistance is due to the presence of certain mobile genetic elements (MGEs), namely the Staphylococcal cassette chromosome mec (SSCmec), which contains the mecA gene encoding a penicillin-binding protein, $\mathrm{PBP} 2 \mathrm{a}$, which has decreased affinity for methicillin compared to other PBPs. ${ }^{3}$

In S.epidermidis, $10 \quad$ different SCCmec structures have been identified, with the short SCCmec type IV element being the most abundant (36\%). 3

In addition to this, biofilm plays an important role in contributing to drug resistance as these organisms shows a remarkable ability to form biofilms on indwelling medical devices such as catheters, surgical implants etc.

Bacteria alter their gene expression in response to external stimuli which enables them to switch from planktonic to biofilm growth mode.

Polysaccharide inter-cellular adhesin (PIA) and capsular polysaccharide adhesion (PSA) plays an important role in the pathogenesis of S.epidermidis as it mediates the contact of bacterial cells with each other, resulting in the accumulation of a multi-layered biofilm. Initial adherence of the bacteria to the surface is mediated by PSA and accumulation of cells is due to presence of PIA. $^{5}$

It has been shown that bacteria within these biofilms can easily exchange genetic information among them and this results in the transfer of antibiotic resistant genes thus contributing to increased resistance. ${ }^{6}$
This serious S.epidermidis infection especially in bloodstream in IPD patients can be prevented if screening of patients and health care staff is done regularly, disinfection of surfaces and devices are done properly, proper hand hygiene by health care staff and antibiotic policy is maintained. treatment should be given after proper sensitivity testing and MIC of drug should be calculated if strain is biofilm producer

Thus considering the high incidence in hospital this study was undertaken to understand the nature of MRSE, resistance patterns and their prevention and treatment.

\section{Materials and Methods}

This was a cross-sectional study conducted at HAHC Hospital, Department of Microbiology, Jamia Hamdard, New Delhi for a period of 6 months.

Ethical clearance: the study protocol was approved by the ethics committee of the institute.

Sample collection: $2.5-4 \mathrm{ml}$ blood from pediatric patients and $8-10 \mathrm{ml}$ blood from adults was collected aseptically in suitable blood culture bottles.

Inclusion criteria: all the blood culture bottles received in lab were incubated in BacT Alert and the positive culture bottle, showing gram positive cocci in smear were requested for one more blood sample preferably from other hand to confirm the organism within 5 day period. Also apart from positive blood culture other parameters were taken into consideration which included: body temperature $>38^{\circ} \mathrm{C}$ or $<36^{\circ} \mathrm{C}$, systolic blood pressure of $<90 \mathrm{mmHg}$, total leucocyte count of $>12,000 / \mu \mathrm{L}$ or $<2000 / \mu \mathrm{L}$ and presence of $>10 \%$ immature neutrophil granulocytes. 
Sample size: the sample size was calculated using the formula $-Z^{2} x p x(1-p) / d^{2}$

$\mathrm{Z}=$ standard deviation, usually taken as 1.96

$\mathrm{P}=$ proportion of the target population expected to have the particular character. (X\%/100)

$\mathrm{d}=$ degree of accuracy, usually set at 0.05 level

Processing of blood sample: This was done using BacT/alert 3D, Biomeriux, the system provides proper incubating temperature and allow the bacteria present in the blood to grow and detection is based on colorimetry technique, detecting color change at bottom of the bottle, this happens due the $\mathrm{CO} 2$ production by the bacteria in the bottle.

Isolation of Staphylococcal epidermidis isolates from the sample were characterized and identified by standard microbiological techniques including gram staining, growth on blood and MacConkey agar, catalase test, coagulase test, sugar fermentation tests, urease test, and novobiocin susceptibility. ${ }^{7}$

Antibiotic susceptibility test - antibiotic susceptibility pattern of all isolates was determined by Kirby-Bauer disc diffusion method and Vitek 2 identification system and results were interpreted as per CLSI guidelines 2019 .

S.aureus ATCC 29213 and E.coli ATCC 25923 were used as positive and negative controls.

Isolates that were oxacillin resistant were further confirmed by cefoxitin disc susceptibility testing and isolates with zone size $\leq 24 \mathrm{~mm}$ were considered as methicillin resistant.

Biofilm production - it was determined by microtitre plate adherence method. ${ }^{8}$
Each isolate of MRSE were mixed in Tryptic Soy Broth (TSB) (HiMedia, Mumbai) and incubated overnight at $37^{\circ} \mathrm{C}$.

The broth was diluted 1:100 and $200 \mu \mathrm{L}$ was pipetted into sterile flat-bottomed 96-well microtitre plates (Tarsons, Kolkata) in triplicate.

Un-inoculated BHI broth was put in eight wells per tray as negative control. Plate was then incubated at $37^{\circ} \mathrm{C}$ for 24 hours.

Post incubation the broth was carefully taken out of the well with the help of micropipette.

The wells were then washed thrice with 200 $\mu 1$ phosphate-buffered saline and kept at $60^{\circ} \mathrm{C}$ for 1 hour.

6) The biofilms were then stained with $200 \mu 1$ of $1 \%$ aqueous crystal violet solution for 1530 minutes, and then washed with phosphatebuffered saline to remove the excess stain.

The plate was then kept inverted for drying. $200 \mu \mathrm{l}$ of ethanol-acetone (80:20 mixture) was added to each well for solubilizing the bound crystal violet.

Absorbance was measured at $570 \mathrm{~nm}$ by using a microtitre plate reader (MAGO-4, Transasia, India).

Cutoff was calculated as mean OD negative control added to 3 times the Standard deviation. (Nc Mean OD + 3xSD).

OD values above the cutoff were considered positive for Biofilm production. The positive isolates were categorized as weak biofilm producer (OD $>$ cut off, $\leq 2 \times$ cutoff), moderate biofilm producer (OD $>2 \times$ cutoff, $\leq 4 \times$ cutoff) or strong biofilm producer (OD $\geq 4$ x cutoff). 


\section{Results and Discussion}

Out of the total 50 Staphylococcus epidermidis samples, 24 were found to be methicillin resistant and the other 26 were methicillin sensitive. The antibiotic susceptibility pattern of methicillin resistant and sensitive strains was determined. The MRSE strains showed high level of resistance to other classes of antibiotics as compared to MSSE strains.

Out of 24 methicillin resistant Staphylococcus epidermidis isolates, 10 isolates were found to show biofilm forming ability accounting for approximately $(41.6 \%)$ and the rest 14 isolates were non biofilm producing (58.3\%).
Among the 10 biofilm producing isolates, 7 were high biofilm producer and the rest 3 were low biofilm producers.

Coagulase-negative Staphylococci were considered as contaminants of bacterial cultures. However, this group especially $S$. epidermidis has emerged as an important opportunistic pathogen of nosocomial infections. The presence of S.epidermidis in the blood originates from dispersal of it from biofilms on indwelling devices, can cause acute sepsis. Bacteremia is associated with healthcare costs, morbidity, and mortality. Immunocompromised and premature neonates are the most vulnerable to $S$. epidermidis in hospitals (Fig. 1 and 2; Table 1 and 2). ${ }^{7}$

Table.1 Prevalence of MRSE and MSSE

\begin{tabular}{|c|c|c|c|}
\hline Strain & MRSE (n) & MSSE (n) & Total Strains (N) \\
\hline $\begin{array}{c}\text { No.of Samples } \\
\text { Percentage \% } \\
\text { (n/N*100) }\end{array}$ & 24 & 26 & 50 \\
\hline
\end{tabular}

Table.2 Biofilm formation by MRSE

\begin{tabular}{|c|c|c|}
\hline Biofilm production & No. of strains (n) & Percentage (\%) $\left(\mathbf{n} / \mathbf{N}^{*} \mathbf{1 0 0}\right)$ \\
\hline High production & 7 & 29.1 \\
\hline Moderate production & 3 & 12.5 \\
\hline No production & 14 & 58.3 \\
\hline
\end{tabular}

Fig.1

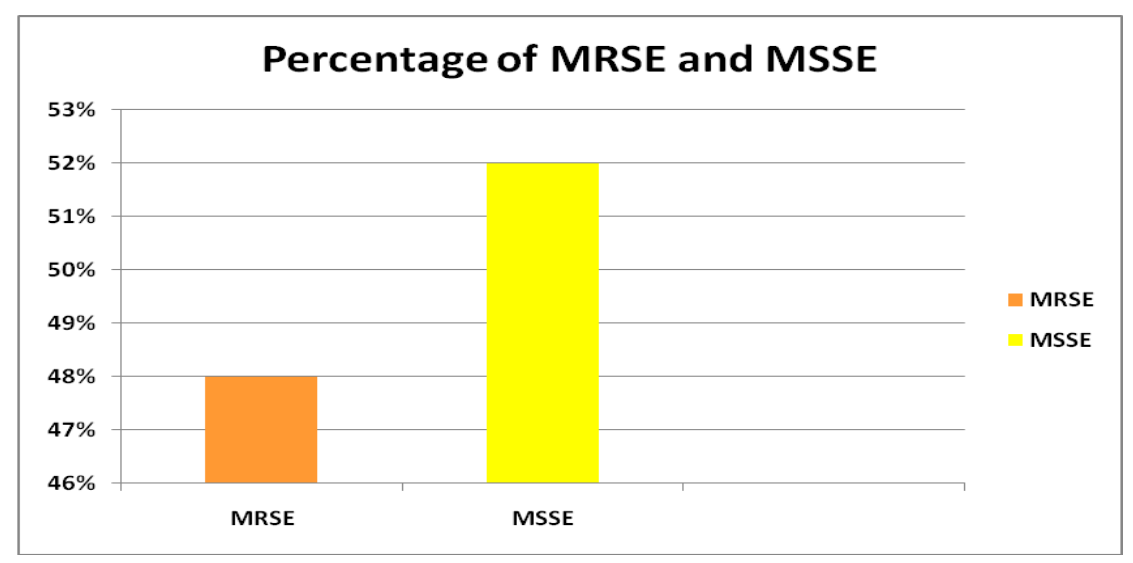




\section{Fig.2}

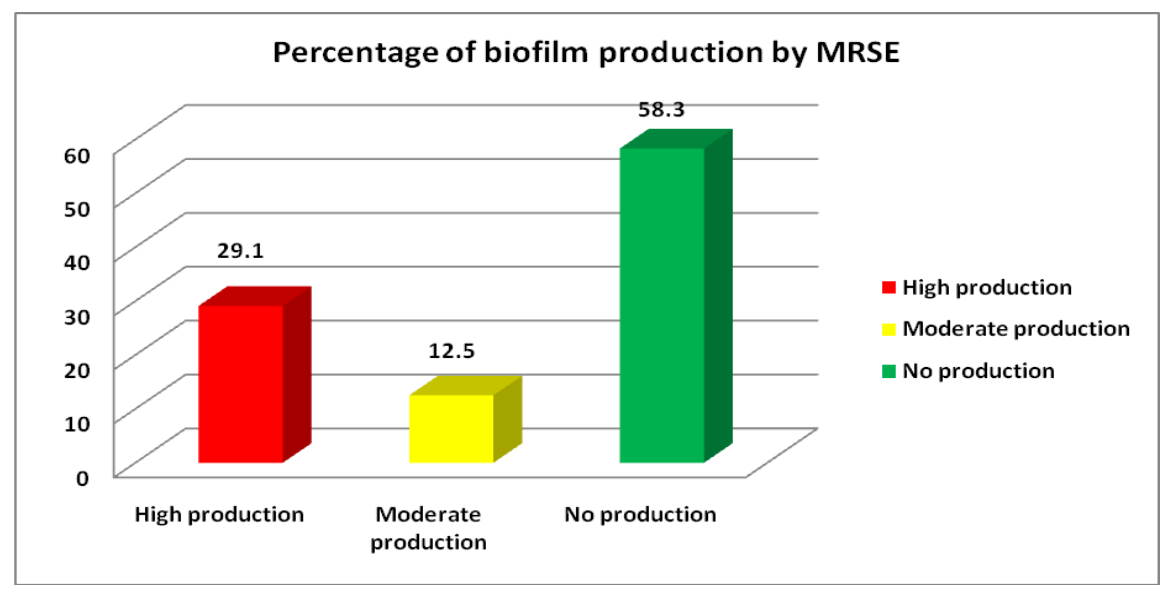

Antimicrobial resistance is one of the most important factors to be determined as increase in antimicrobial resistance leaves us with very limited treatment options. In accordance with many authors ${ }^{910}$ higher resistance rates were observed in MRSE isolates as compared to MSSE against all antimicrobials tested.

In our study all MRSE isolates were resistant to benzylpenicilin. An overall high prevalence of resistance for non beta-lactam antimicrobials was observed including Erythromycin (95.8\%), Clindamycin (54.1\%), Ciprofloxacin $(50 \%)$. Only one isolate was found to be resistant to linezolid (4.16\%). However certain studies show that there is a steady increase in resistance to linezolid which is generally seen in critically ill patients or patients with prior exposure to linezolid; however linezolid resistance in staphylococcal isolates is seen rarely in India ${ }^{11}$.This could be accounted to its infrequent use in India. Still linezolid resistance is a matter of concern as resistance is plasmid mediated it can be transmitted to susceptible strains and thus needs to be properly screened. Also linezolid resistance leaves us with limited treatment options such as glycopeptides and daptomycin.

No isolate was found to be resistant to vancomycin, teicoplanin, daptomycin in our study suggesting the use of these drugs in therapy, however these drugs need to be administered only after proper susceptibility testing so as to prevent overuse of these antibiotics and development of resistance. Although certain studies have reported resistance again vancomycin. However the data is inadequate and requires more insight into it.

On the other hand, in MSSE maximum resistance was noted against Erythromycin (61.5\%) and Benzylpenicillin (34.6\%). All other antibiotics show little or no resistance which is in concordance with the study conducted by Koksal et al., ${ }^{9}$.

Our findings suggest that better antibiotic combinations should be selected based on locally prevalent antibiogram patterns for better management of these infections. Furthermore, antibiotics should be used judiciously for optimal treatment of infections and prevention of selection pressure mediated drug resistance.

The high level of antimicrobial resistance seen in S.epidermidis can be accounted to their ability to form biofilms. Biofilm production plays an important role in establishing an infection on catheter related devices and acts as a virulence factor. Biofilm 
production masks the bacteria during conditions of stress and high antimicrobial concentration thus conferring resistance to the bacteria and making such infections difficult to treat. In our study, 24 isolates of MRSE were tested for biofilm formation. The test was performed using microtiter plate assay as it helps in differentiating between weak and strong biofilm producers.

Out of the 24 MRSE isolates tested, only 10 were found to produce biofilm accounting for $41.6 \%$, the rest 14 were non biofilm producers $(58.3 \%)$. Among the biofilm producers, only 7 were high biofilm producers $(29.1 \%)$ and 3 were moderate biofilm producers (12.5\%). Similar results were obtained by Agarwal and Jain ${ }^{11}$ where they showed that biofilm production was seen in $38.2 \%$ of MRSE.

Thus biofilm production depicts a major concern and requires further research in this area to develop novel therapeutic intervention. Furthermore apart from focusing on antibiotic prophylaxis, preventive strategies should be taken into account to control S.epidermidis mediated biofilm production on catheter related devices.

Although this study highlights the growing concern in the area of microbiology; it presents with certain limitations as well.

This includes the small sample size due to the short period of study. No use of multiple phenotypic methods for detecting methicillin resistance and biofilm production which otherwise would have increased the sensitivity of the results. The study lacked in the use of molecular methods such as PCR for the detection of genes responsible for methicillin resistance and biofilm formation.

In conclusion to the best of our knowledge, this is the first study conducted in Delhi correlating the antimicrobial resistance pattern and biofilm production among the Staphylococcus epidermidis strains. This study advocated the high resistance pattern among S.epidermidis strains which needs to be controlled as they can transmit drug resistant genes to other organisms such as S.aureus. Also the use of catheter related devices should be carefully monitored.

Although more advanced and sensitive molecular methods are to be employed to determine the common clones/strains of hospital and community acquired S.epidermidis in order to control or at least delay the future emergence of S.epidermidis as potential pathogen.

It is also recommended to strictly adhere to the infection control guidelines to prevent the spread of Staphylococcus epidermidis infections in hospitals and tracking of drug resistant colonization through active surveillance in high risk units.

We hope that this study will form a useful reference for clinical microbiologist, physicians and others attempting to monitor the prevalence of MRSE infection and for the treatment of patient with such infection, and this study will definitely add on valuable information regarding MRSE in North India to the existing data.

\section{References}

1. Kloos WE, Banneran TL. Update on clinical significance of CONS. Clinical Microbial Review 1994; 7: 117-40.

2. Garrett DO, Jochimsen E, Murfitt K, Hill B, McAllister S, Nelson P, Spera RV, Sall RK, Tenover FC, Johnston J, Zimmer B. The emergence of decreased susceptibility to vancomycin in Staphylococcus epidermidis. Infection Control \& 
Hospital Epidemiology. 1999 Mar; 20(3): 167-70.

3. Lai Y, Cogen AL, Radek KA, Park HJ, MacLeod DT, Leichtle A, Ryan AF, Di Nardo A, Gallo RL. Activation of TLR2 by a small molecule produced by Staphylococcus epidermidis increases antimicrobial defense against bacterial skin infections. Journal of Investigative Dermatology. 2010 Sep 1; 130(9): 2211-21.

4. Heilmann C, Schweitzer O, Gerke C, Vanittanakom N, Mack D, Götz F. Molecular basis of intercellular adhesion in the biofilm- forming Staphylococcus epidermidis. Molecular microbiology. 1996 Jun; 20(5): 1083-91.

5. Mack D, Davies AP, Harris LG, Rohde H, Horstkotte MA, Knobloch JK. Microbial interactions in Staphylococcus epidermidis biofilms. Analytical and bioanalytical chemistry. 2007 Jan 1; 387(2): 399408.

6. Arciola CR, Campoccia D, Gamberini S, Donati ME, Pirini V, Visai L, Speziale P, Montanaro L. Antibiotic resistance in exopolysaccharideforming Staphylococcus epidermidis clinical isolates from orthopaedic implant infections. Biomaterials. 2005 Nov 1; 26(33): 6530-5.
7. Collee JG, Miles R, Watt B. Tests for identification of bacteria. Mackie and McCartney practical medical microbiology. 1996; 14: 131-49

8. Stepanovic S, Vukovic D, Hola V, Bonaventura GD, Djukic S, Irkovic IC, et al., Quantification of biofilm in microtitre plates: overview of testing conditions and practical recommendations for assessment of biofilm production by Staphylococci. APMIS. 2007; 115: 891-99.

9. Koksal F, Yasar H, Samasti M. Antibiotic resistance patterns of coagulase-negative staphylococcus strains isolated from blood cultures of septicemic patients in Turkey. Microbiological research. 2009 Jan 1; 164(4): 404-10.

10. Singh S, Dhawan B, Kapil A, Kabra SK, Suri A, Sreenivas V, Das BK. Coagulase-negative staphylococci causing blood stream infection at an Indian tertiary care hospital: prevalence, antimicrobial resistance and molecular characterisation. Indian journal of medical microbiology. 2016 Oct 1; 34(4): 500

11. Agarwal A, Jain A. Association between drug resistance \& production of biofilm in staphylococci. The Indian journal of medical research. 2012 Apr; 135(4): 562.

\section{How to cite this article:}

Maryam J. Ansari, Rachna Tewari and Mridu Dudeja. 2020. Methicillin Resistant Staphylococcus epidermidis in Blood Samples-Can its Spread be Prevented in Hospitals? Int.J.Curr.Microbiol.App.Sci. 9(09): 542-548. doi: https://doi.org/10.20546/ijcmas.2020.909.069 